\title{
Procesos Emergentes Que Identifican El Desarrollo De La Inteligencia Emocional A Través De La Resolución De Problemas En La Educación Superior
}

\author{
Luisa Morales-Maure, Maestría \\ Orlando García-Marimón, Maestría \\ Universidad de Panamá/ Departamento de Matemática \\ Marcos Campos Nava, Maestría \\ Universidad Autónoma del Estado de Hidalgo/ \\ Área Académica de Matemáticas y Física
}

doi: 10.19044/esj.2016.v12n1p411 URL:http://dx.doi.org/10.19044/esj.2016.v12n1p411

\begin{abstract}
The didactic approach is based on the premise that the training of mathematics teachers must include, as a minimum, the knowledge of three fundamental areas, closely related to each other: mathematics, epistemology and didactics. The key element to distinguish the knowledge base for teaching can be found at the intersection between mathematics and didactics, in the capacity of a teacher to transform knowledge and discipline in a didactically impressive way, adapting and developing it to the different abilities and capacitates students possess. This is the general principle of this article, which aims to analyze the emotional intelligence profile of a group of higher education professors specialized in the teaching of mathematics who work in two educational institutions in Panama. The study used a descriptive-correlational methodology, not experimental, which permitted an approach to the different constructs of the professors' emotional intelligence through the solving of mathematics problems and their performance in the higher education field. Nowadays, there are not many profound studies on this topic that could permit us identify the emotional intelligence of teachers to teach and foster mathematics competence in the classroom.
\end{abstract}

Keywords: Aptitude, ability, cognition, learning process, emotional mathematics, mathematics teacher training

\section{Resumen}

La aproximación didáctica parte de la premisa de que la formación de los profesores de matemáticas debe incluir, como mínimo, conocimientos de tres áreas fundamentales que son matemáticas, epistemología y didáctica, las 
cuales deben estar estrechamente relacionadas entre sí y con su praxis. La clave para distinguir el conocimiento base para la enseñanza está en la intersección entre las matemáticas y la didáctica, en la capacidad de un profesor para transformar su conocimiento disciplinar en formas que sean didácticamente impactantes y adaptables a la variedad de habilidades y recursos que poseen los estudiantes. Este es el principio de este artículo, que tiene como objetivo general analizar el perfil de inteligencia emocional de una muestra de docentes de educación superior especialistas en matemática vinculados a dos instituciones educativas en Panamá. La metodología empleada fue descriptiva correlacional no experimental. Lo cual permitió un acercamiento a los diferentes constructos de la inteligencia emocional del docente a través de la resolución de problemas matemáticos y su desempeño docente en la educación superior. En la actualidad existen pocos estudios a profundidad sobre el tema, que permitan identificar la inteligencia emocional del docente para saber enseñar y potenciar en sus estudiantes competencias matemáticas en el salón de clases.

Palabras clave: Aptitud, habilidad, cognición, proceso de aprendizaje, inteligencia emocional, formación de profesores de matemáticas

\section{Introducción}

La estrecha relación entre lo emotivo, cognitivo y los procesos de aprendizaje, la toma de decisiones y la memoria se conoce bien a partir de los estudios realizados en el campo de la psicología experimental a lo largo de su historia. Esto ha generado interés en diversas comunidades académicas, llegando a considerarse un factor predictivo para el alcance de las metas personales y académicas en los seres humanos. En los últimos años, algunas pruebas experimentales indican que estos procesos cognitivos pueden verse afectados si las áreas del cerebro responsable de las emociones están deterioradas; por lo tanto, se puede argumentar que la emoción es uno de los componentes de la inteligencia (Goleman, 1995). De este modo, la inteligencia se puede entender como la capacidad de adaptación a un medio cambiante. Una parte de la problemática escolar no radica en la capacidad o incapacidad de los alumnos para comprender determinados temas, sino en su actitud hacia la escuela, el profesor y la asignatura. Autores proponen que el éxito de un alumno en clase de Matemática, está en relación con la actitud positiva hacia la actividad que realice de esta materia y que se incluyen rasgos de personalidad que involucran las esferas intelectual y emocional.

Se ha podido observar el poco interés, los fracasos constantes y poco desarrollo matemático de los estudiantes en Panamá. Esto se sustenta en que la mayoría de los estudiantes del nivel medio superior adolecen de conocimientos matemáticos básicos, observándose que los estudiantes que 
egresan de instituciones oficiales $\mathrm{y}$ en menor medida de escuelas particulares, tienen conocimientos insuficientes en el ámbito del razonamiento matemático (Maure, L. M., \& Marimón, O. G., 2014). La calidad de la enseñanza es uno de los principales problemas que enfrenta hoy el sistema educativo panameño, tras las deficiencias detectadas en asignaturas como español, matemática y ciencias naturales.

El reciente Tercer Estudio Regional Comparativo y Explicativo realizado en 15 países de América Latina y el Caribe, entre ellos Panamá, reveló el bajo nivel de la educación en la nación istmeña. Este estudio contempló pruebas académicas en escuelas públicas del país, en las cuales los resultados estuvieron por debajo de los 700 puntos adoptados como promedio para evaluar a Latinoamérica.

Es evidente que existe una gran problemática en el aprendizaje de la Matemática ya que cada año se refleja un creciente número de fracasos estudiantiles (relacionados con esta asignatura), en cualquier parte del mundo. La directora de Evaluación Educativa del Ministerio de Educación de Panamá, indicó que a partir de este estudio los esfuerzos deben centrarse en mejorar el desempeño del profesor de matemática y facilitar las estrategias a los estudiantes para la resolución de problemas.

Los retos actuales de la docencia en la educación superior están ligados a procesos de acreditación que obligan cada vez más al mejoramiento continuo en lo que conocemos hoy como la acreditación institucional, proceso para reconocer o certificar la calidad de una institución o de un programa educativo que se basa en una evaluación previa de los mismos (CONEAUPA). Con una visión holística, todos los factores son importantes pero, sin duda, el docente establece la diferencia en la mejora de su clase.

\section{Marco teórico}

El docente es responsable del desarrollo de todo un grupo de estudiantes. La enseñanza no se trata sólo de la difusión del conocimiento matemático, ya que los docentes también deben absorber en la vida cotidiana lo que piensan sus estudiantes. Este estudio se centra en la inteligencia emocional de los profesores de matemáticas durante el proceso de enseñanza superior.

\section{Desempeño Docente}

El rendimiento es básicamente la capacidad del individuo para utilizar sus conocimientos de manera eficiente y eficaz. Cuando se analizó el desempeño del profesor, se basaba tanto en el aspecto intelectual y físico. En este contexto, la creación de conocimiento está en constante evolución y sometida permanentemente a cambios relacionados con el alud de informaciones y de nuevos conocimientos que recibimos de las tecnologías 
de la información y de la comunicación (TIC). La tierra se nos ha hecho pequeña, conocemos inmediatamente qué pasa en un rincón alejado del planeta y quizás desconocemos qué pasa en casa de nuestro vecino. Esta situación nos obliga a replantear el papel de la escuela del futuro y el perfil que debe tener el profesorado del siglo XXI (Travería, G.T., 2008, 185-186). En relación con el rendimiento, también identificó el papel de los profesores en diferentes áreas como habilidad de trabajo, actitud hacia el trabajo y la participación en su desarrollo profesional. Brophy y Good (1986) presentan un enfoque atractivo para la evaluación del desempeño docente, incluye la socialización de los estudiantes y el desarrollo personal del alumno en el desempeño de los profesores universitarios.

Es indispensable establecer criterios claros sobre los procesos emergentes del docente de matemática en la educación superior, que tiene como obligación ocuparse de enseñar a sus alumnos a aprender y a tomar iniciativas. En el mismo, enfatizar la actuación y mejora de sus competencias pedagógicas mediante programas adecuados de formación continua que estimulen la innovación permanente en los programas de estudio y los métodos de enseñanza-aprendizaje y ser capaz de comprender y desarrollar los cuatro pilares de la educación en la era actual con lo que cada uno de ellos implica: Saber conocer, saber hacer, saber estar y saber aprender. En la figura 1, se exponen las directrices que representa cada pilar del saber según lo establecido por la UNESCO:

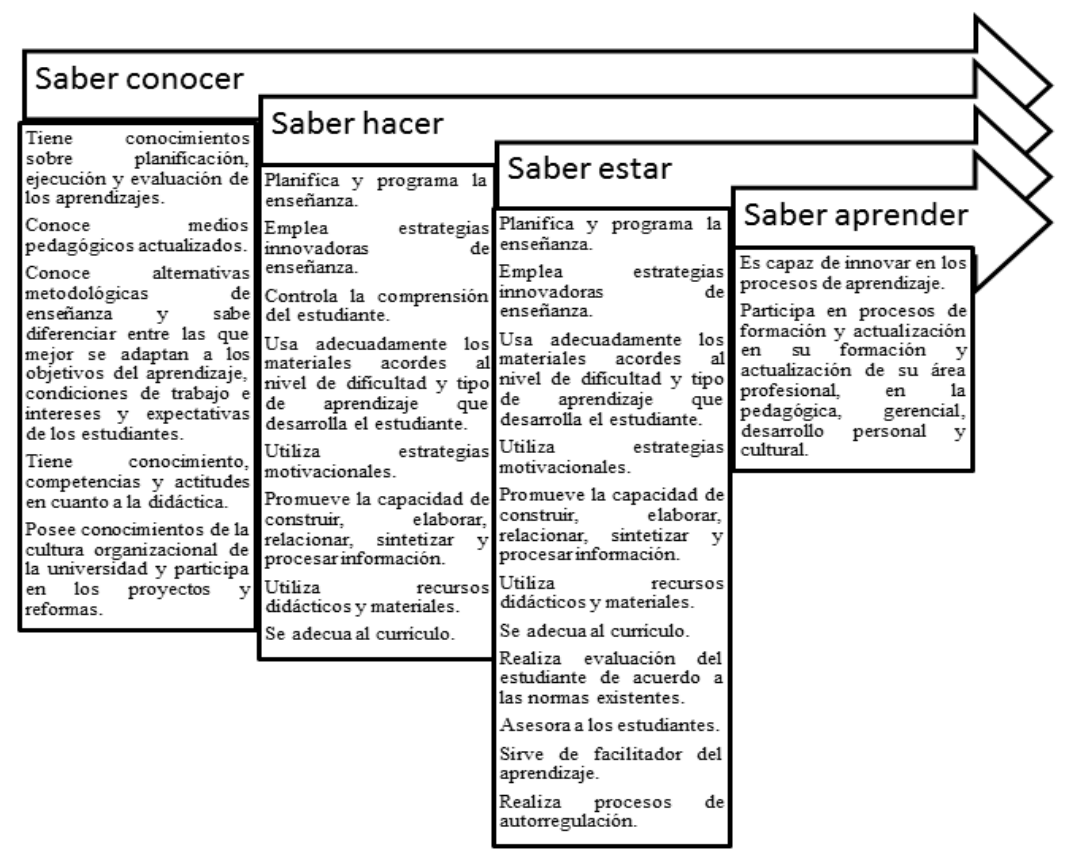

Figura 1 Pilares del Saber, Delors, J (1996). 
Todo lo anterior permite establecer que la formación del docente debe responder a un perfil y a unas funciones que destaquen que no sólo se base en la transmisión del conocimiento sino también en orientar al estudiante hacia al aprender a aprender, el análisis, la crítica y la creatividad constante que les permita ser emprendedores, y además, debe involucrarse en los procesos de investigación.

En este sentido, dirigir un curso de formación para profesores es de naturaleza muy diferente a dirigir un curso para estudiantes, en principio por el tiempo que se puede dedicar: mientras que un curso de formación por lo general es de duración corta, (medido en horas); el curso escolarizado es de duración mayor, (se suele medir en semanas).

Por otro lado, tratar de educar a personas adultas (con una trayectoria académica, profesional y laboral consolidada), puede ser de naturaleza muy distinta a educar a adolescentes o jóvenes universitarios, en buena medida por el auto concepto que éstos ya se han formado.

Al cuestionarse sobre la naturaleza que deben tener los cursos de formación para profesores de matemáticas de nivel medio superior y universitario del sistema educativo panameño, que les permita desarrollar su inteligencia emocional, para que a su vez esto repercuta en su práctica docente, una pregunta natural es ¿qué principios pueden ser útiles a quien diseñe e imparta dichos cursos para llevar un proceso de formación con profesores de matemáticas?

En el ámbito de la formación y capacitación de personal, el término "andragogía” se utiliza para identificar a la educación centrada en los adultos; desde ese enfoque, se menciona que la "andragogía" es un cuerpo de conocimientos y métodos, encaminados para facilitar su educación.

El hombre, al ser perfectible, requiere de un proceso educativo permanente que lo capacite para actualizar sus potenciales, de ahí que surja la andragogía, que es una disciplina que nos obliga a preocuparnos de la educación del adulto de un modo especial y planificado. (Red Tercer Milenio, p. 44)

Desde la perspectiva de algunos investigadores, la educación centrada en el adulto, debe ser planificada diferente a la educación centrada en niños y jóvenes, en otras palabras, al estar dirigiendo un proceso de formación o capacitación para personas adultas, el instructor debe utilizar principios diferentes a los que se utilizan en la pedagogía.

El concepto andragogía es un neologismo propuesto por la UNESCO, para designar la ciencia de educación para adultos [...] es un enfoque teórico de la educación de adultos que se sustenta en principios [...] (Maya et al, 2001, p.87). 
En este sentido, algunos autores como Knowles (citado por Montoya \& Velázquez, sin año de publicación), consideran a la educación para adultos centrada en seis principios que a continuación se presentan:

Su necesidad de conocer

Su concepto personal (autoconcepto)

Su experiencia previa

Su disposición para aprender

Su inclinación al aprendizaje

Su motivación para aprender

Montoya \& Velázquez mencionan también que Malcom Knowles, fue quien hizo popular el concepto "Andragogía”, el adulto es auto-dirigido en su aprendizaje, además cuenta con un importante recipiente de información gracias a la experiencia. El aprendizaje está dirigido hacia solución de problemas, la motivación de aprender es interna

Por otra parte Mezirow (citado por Montoya \& Velázquez, sin año de publicación), considera el aprendizaje en el adulto como un aprendizaje transformativo donde plantea que usando la reflexión crítica junto con el pensamiento crítico la persona puede cambiar sus marcos de referencia, creando nuevos modelos de pensamiento que guíen nuestra acción. Es decir se plantea que aprendemos cuando realmente tenemos conciencia de todos los elementos que afectan nuestras decisiones.

¿Pueden estos factores tener relación con la resistencia a utilizar nuevas tecnologías en su práctica docente?

Por otro lado, aunado al autoconcepto que los profesores de matemáticas se han formado, la gestión en el salón de clase es también un aspecto importante de la actuación del profesor en clase. Se relaciona con la manera en que el docente lleva un ambiente productivo en clase. Berliner (1986) describió la gestión del aula como requisito previo a la enseñanza efectiva. En la gestión del entorno el profesor debe observar la clase críticamente, desarrollar reglas para los estudiantes de acuerdo a los comportamientos y requerimiento del objetivo docente.

La efectividad del docente también tiene que ver con centrarse en las diferencias individuales. La razón es que el comportamiento de todos los seres humanos es cambiante, por lo que también es responsabilidad del maestro para centrarse en los comportamientos de sus estudiantes. Por lo tanto, la atención individual también se considera el aspecto importante del rendimiento en el proceso de enseñanza. Brophy (1983) se centró en el uso sostenido de herramientas de motivación en clase, así, también es un aspecto de la actuación docente. Los profesores también juegan un papel en la prestación de la guía a los estudiantes en sus problemas académicos. Para proveer la guía para el estudiante es también el aspecto importante del desempeño del profesor. 
Los profesores deben saber, que es lo que está enseñando, y lo que es enseñar. Debe tener la capacidad de presentar el tema de una manera dinámica, pues, en consecuencia de ello, se tiene el buen control de la clase. Es por ello que para medir el desempeño del docente se tomaron en cuenta los siguientes aspectos:

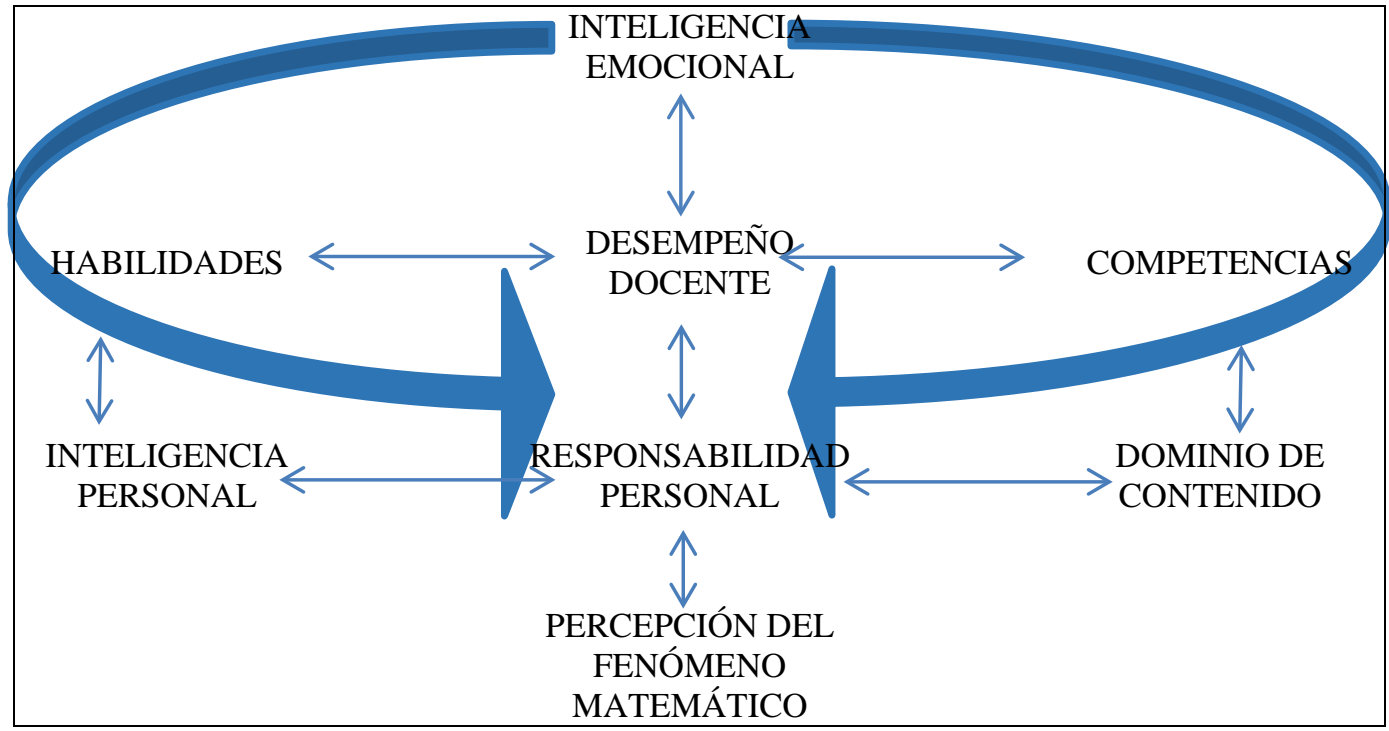

Figura 2 Relaciones generadas desde el conocimiento matemático en docentes universitarios

\section{Inteligencia emocional}

Hay dos escuelas de pensamiento acerca de la inteligencia emocional (IE) en la literatura. Se proponen dos modelos generales: Una de ellas es el modelo basado en la habilidad propuesto por Mayer y Salovey (1997). Algunos otros enfoques son también propuesto por Baron (1997), Furnhan, A., Petrides (2003), Shutte, Malouff, Hall, Haggerty, Cobre, Godlen, CJ, Dornheim (1998).

La IE ayuda a una persona en situaciones difíciles en las que se debe actuar de acuerdo a la demanda de la época (Mayer, J.D., Salovey, P., y Caruso, D., 2002). La inteligencia emocional es una mezcla de capacidades que incorpora la posibilidad de discernir los sentimientos en sí mismo y en otras personas, utilizar los sentimientos para mejorar la ejecución, comprender los sentimientos e información entusiasta, y sentimientos directos en el mismo y en otras personas (Mayer y Salovey, 1997).

Bisquerra, $(2007,27)$ consideraba que la "emoción es un estado complejo del organismo caracterizado por una excitación o perturbación que predispone a una respuesta organizada. Las emociones se generan como respuesta a un acontecimiento externo o interno.” Requiere abordar los sentimientos en un estado sostenido durante un lapso de tiempo LeDoux 
(1996) y Frijda (1994), establecieron que las emociones permiten identificar los hechos relevantes para el ser humano.

El concepto de inteligencia emocional (IE) incluye factores como: afectivos, emocionales, personales y sociales. Además, Mayer y Salovey (1997, 12), modificaron esta noción en relación a la "habilidad de percibir con exactitud, valorar y expresar emociones; la habilidad de acceder o generar sentimientos que faciliten el pensamiento; la comprensión emocional y conocimiento emocional; habilidad de regular emociones para promover el crecimiento intelectual y emocional” prolongando así los trabajos de Wechsler (1940) y Gardner (1983).

Shapiro (1997), consideró que la inteligencia emocional se relaciona con las cualidades emocionales que conducen al éxito. Algunas de ellas son: la empatía, expresión y comprensión de los sentimientos y la capacidad de adaptación. Estas cualidades contribuyen a que el individuo pueda resolver problemas entre pares y en comunidad en forma creativa. Para Cooper y Sawaf (1997), la inteligencia emocional es una aptitud para captar emociones. Sin embargo, para Simmons y Simmons (1997), esta es un conjunto de necesidades desde lo emocional, que orienta las conductas visibles.

En el campo de la Matemática, autores como Polya (2001) han declarado, que sería un error el creer que la solución de un problema es un asunto puramente intelectual ya que la determinación y las emociones juegan un papel importante (p. 80). Halmos, (citado en Gómez-Chacón, 2000, p. 15) menciona ¿Son las Matemáticas algo emocional? La gente suele decir que no, pero yo creo que sí lo son. Un matemático es una persona y tiende a sentir emociones fuertes sobre qué parte de las Matemáticas está dispuesto a soportar y, naturalmente, emociones fuertes sobre otras personas y las clases de Matemáticas que les gustan. Por ejemplo: ¿qué prefieres, números o dibujos, símbolos o gráficas, álgebra o geometría? Yo soy principalmente un hombre de números, y no sólo me ponen nervioso los dibujos, sino incluso la gente que los prefiere.

Al igual que estos autores, pensamos que el estilo matemático está relacionado con las emociones. Eso quiere decir que los referentes afectivos tales como: las emociones, las creencias o las actitudes no representan algo artificial, sino que están comprometidos e involucrados con el éxito o el fracaso de los estudiantes y de los docentes en el desarrollo de sus tareas destinadas a la transposición de conocimientos, así como de la construcción de saberes matemáticos en los alumnos.

En este sentido, tanto los docentes como los estudiantes podrían ser responsables de los bloqueos (de tipo emocional), que se presentan en el aprendizaje de contenidos matemáticos. Incluso, Gómez-Chacón (2002) señala que "la insuficiente comprensión de los contenidos puede ser producto 
de sentimientos de desconcierto y perplejidad”. También indica que los sentimientos de aburrimiento pueden codificar la ausencia de compromisos. De manera que cuando se habla de miedo, aburrimiento, desconcierto, desamor, disgusto, rabia y desilusión hacia la Matemática, existe una introspección en los alumnos, que se traduce a una expectativa de fracaso, en las tareas destinadas a aprender esta ciencia y, por ende, se configuran actitudes desfavorables hacia esta asignatura.

Las creencias relativas al autoconcepto del estudiante como aprendiz de Matemática es, según Gómez-Chacón (2000), una de las variables que más influyen en el aprendizaje y en la enseñanza de esta asignatura la cual está relacionada con sus actitudes, su perspectiva del mundo matemático y con su identidad social. Los elementos más importantes de este constructo son los conocimientos subjetivos y las emociones referidas a los siguientes aspectos: el interés en Matemática, la eficiencia, la motivación y el placer que causa está la atribución causal de éxito o fracaso escolar y el autoconcepto como miembro de un determinado grupo social.

\section{Metodología}

Para acercarnos a cumplir el objetivo propuesto "analizar el perfil de inteligencia emocional de una muestra de docentes de educación superior especialistas en matemática” se trazó el siguiente diseño metodológico:

Este estudio se realizó en la Universidad de Panamá, campus Harmodio Arias Madrid localizado en la ciudad de Panamá, se estudió a un grupo de 25 docentes. Se consideró como población a las escuelas básicas urbanas y rurales de Los Santos, Coclé y Panamá. Con profesores de formación general, con y sin especialización en matemática, pero que impartían sus clases en los niveles medio y superior. Por la naturaleza del proyecto, donde interesa el proceso y el producto (Godino, 2003), el tipo de estudio es descriptivo de enfoque cuantitativo pues se recolectaron datos sobre diferentes aspectos de los procesos emergentes de los docentes que se desarrolló durante el periodo escolar de marzo a diciembre, en el transcurso del año 2014-2015.

La investigación descriptiva busca especificar propiedades, características y rasgos importantes de cualquier fenómeno que se analice (Hernández, Fernández y Baptista, 2006).

El diseño de la investigación es de tipo no experimental porque no se puede manipular las variables, los datos a reunir se obtuvieron de los docentes. La investigación no experimental es la que se realiza sin manipular deliberadamente variables; lo que se hace en este tipo de investigación es observar fenómenos tal y como se dan en su contexto natural, para después analizarlos (Hernández, Fernández y Baptista, 2006). 
Se diseñó un curso de perfeccionamiento guiado para profesores de educación media y superior, con la finalidad que construyeran herramientas y recursos conceptuales, metodológicos con y sin la tecnología. Que les permitan desarrollar tareas y ambientes de instrucción que apoyen la construcción de un pensamiento algebraico en los estudiantes con el uso de nuevas tecnologías.

La estrategia didáctica utilizada en cada una de las fases de trabajo, contempló intervenir, por una parte, la totalidad del programa curricular en educación matemática correspondiente, y otra, la dinámica del aula en las escuelas considerando contextualmente y metodológicamente su aplicación.

La primera fase (ya ejecutada), correspondiente al momento de la interacción didáctica en el aula, se centró en las acciones de los profesores en el curso de perfeccionamiento, que se realizó de manera sistemática con clases presenciales durante cuatro horas, en una semana, y contempló el desarrollo de contenidos matemáticos previamente elaborados con base en ejercicios, situaciones problemáticas, y a tipos de competencias matemáticas, de esta fase surgieron principios para la elaboración del trabajo que deseamos continuar perfeccionando.

Al interactuar con los profesores dentro del contexto del aula, buscando incidir en aspectos de tipo afectivo que pudieran modificar el autoconcepto que ellos tienen sobre lo que significa enseñar matemáticas y en consecuencia modificar su praxis, entre otros aspectos incorporando el uso de herramientas digitales (TICs) con sus estudiantes, surgen algunas cuestiones naturalmente ¿Puede influir el autoconcepto de un profesor respecto a lo que para él significa enseñar matemáticas, en el tipo de práctica docente que acostumbre? ¿La actitud del profesor puede ser un factor que favorezca o entorpezca su formación en el uso de nuevas tecnologías?

La necesidad que tiene el docente de matemáticas en reflexionar sobre cómo influyen sus percepciones, creencias y concepciones en su práctica docente es de suma importancia, como mencionan Barrantes y Mora (2008).

Cualquier estrategia a utilizar en el proceso de enseñanza aprendizaje de las matemáticas estará sustentada en alguna percepción sobre la naturaleza de las matemáticas. Esta percepción influirá en el tipo de actividades y ambiente de aprendizaje que el profesor proponga a sus estudiantes para inducir en ellos el aprendizaje de los conceptos matemáticos. De esta manera, según la visión particular acerca de las matemáticas, se puede propiciar en los estudiantes diferentes tipos de aprendizaje que pueden ser memorísticos y algorítmicos o, por el contrario, aprendizaje que requieran del alumno un pensamiento creativo para enunciar conjeturas, aplicar de 
manera razonada la información, descubrir y, en general, construir su conocimiento.

Aunado a la idea que cada profesor tiene sobre lo que significa enseñar matemáticas y la matemática en sí misma, se puede considerar que también su formación cuando fue estudiante tiene repercusiones en su práctica docente actual; si el profesor de matemáticas fue educado bajo un modelo tradicional en el que la tecnología giraba en torno al pizarrón y tiza y él considera que así aprendió matemáticas adecuadamente, podría suponer que enseñar de la misma forma en que aprendió, es un buen método.

Se sabe que existe resistencia entre algunos profesores, incluidos los de matemáticas, para incorporar a su práctica docente a las nuevas tecnologías, como es el caso de calculadorasgraficadoras o computadoras con software especializado, esto puede ocurrir en parte por la instrucción matemática que recibió el profesor en su etapa como estudiante, la cual está ligado con la forma en que concibe a las matemáticas y el cómo las enseña. (Campos, 2010 a, p.73).

Sin embargo, es muy restrictivo considerar que si una práctica funcionó durante años de manera específica, no sea necesario modificarla o mejorarla; se puede afirmar que la mayoría de los profesores son producto de la educación del siglo XX; aunque con modelos educativos del siglo XIX; $\mathrm{y}$ por otro lado, los estudiantes que hoy se atienden, son producto del siglo XXI; los cuáles están influenciados por los avances tecnológicos que se vienen dando en las últimas dos décadas, por lo cual no tiene sentido resistirse a la incorporación de las nuevas tecnologías; la atención se debería centrar a usar estos recursos como una oportunidad para diseñar tareas de aprendizaje que promuevan un mejor entendimiento de algunos conceptos matemáticos.

Esta resistencia al cambio es incomprensible, pues en la vida cotidiana, los profesores de matemáticas hacen uso de todo tipo de tecnologías que no estaban a su alcance hace algunos años, por ejemplo el uso de cajeros automáticos, o enviar mensajes de texto por medio del teléfono celular, lo cual implicó que modificaran sus concepciones y prácticas acerca del manejo de sus finanzas o de la forma de comunicarse con sus semejantes, en este sentido, debería existir una actitud de aceptación por parte del profesor para incorporar nuevas tecnologías a su actividad. (Campos, 2010, a, p.73).

Después de relatar algunos de los aspectos centrales de la primera fase de éste estudio, en el que se tuvo intervención con los profesores dentro de un proceso formativo, considerando algunos de los aspectos de la educación centrada en los adultos “andragogía” para incidir en el autocepto 
de los profesores hacia lo que significa para ellos enseñar matemáticas, haciendo énfasis en la incorporación de las TICs a su práctica docente, se describe ahora lo que corresponde a la segunda fase, la cual consistió de asesorías personalizadas, por una parte para tener un acercamiento a los profesores de aula con un efectivo apoyo a la enseñanza de la matemática, y por otra, un seguimiento de la transferencia de las acciones didácticas a los alumnos involucrados.

De acuerdo a los objetivos del proyecto, se diseñaron, elaboraron y validaron instrumentos de evaluación en matemática, a través del contenido y del juicio de expertos, desarrollados bajo una perspectiva de resolución de ejercicios y problemas de acuerdo a los objetivos fundamentales y contenidos mínimos, lo que permitió probar la significatividad de los aprendizajes logrados tanto a nivel de profesores como de sus alumnos en las escuelas. De manera particular permitió evaluar la competencia matemática de los profesores.

En calidad de pre-test y pos-test fue aplicada a los profesores y sus respectivos alumnos, la prueba de competencia matemática. Estas unidades fueron tratadas en su totalidad durante el perfeccionamiento y de acuerdo a los objetivos del proyecto, la resolución de problemas fue incluida en forma transversal durante toda la ejecución del mismo para este segundo seminario. En igual forma y en calidad de pre-test y post-test, será aplicado a los profesores un instrumento evaluativo basado en el modelo de competencia descrito anteriormente, con la finalidad de medir las competencias consideradas en el estudio. El perfeccionamiento, en su totalidad consideró una concepción de la didáctica de la matemática, que relacionó la enseñanza de los contenidos y la resolución de ejercicios, tipos de problemas y tipos de competencias matemáticas, a fin de acceder de manera contextualizada a los conceptos propuestos en el currículo reformado. Se privilegiaron las acciones centradas en los profesores, originando discusiones al interior de los grupos y proporcionando oportunidades de interacción activa entre ellos. La enseñanza a los profesores se implementó teniendo en consideración una perspectiva constructivista de aprendizaje y fue apoyado por materiales instruccionales, creados por ellos como un medio efectivo para estos fines. Los materiales de instrucción, la utilización de películas y videos exhibidos, se usaron como elementos facilitadores de la tarea en el proceso didáctico, como un medio para ofrecer alternativas de mejoramiento y como un elemento motivador de todo el proceso de enseñanza-aprendizaje. (Figura 3). 
Figura 3 Desarrollo metodológico de los procesos emergentes.

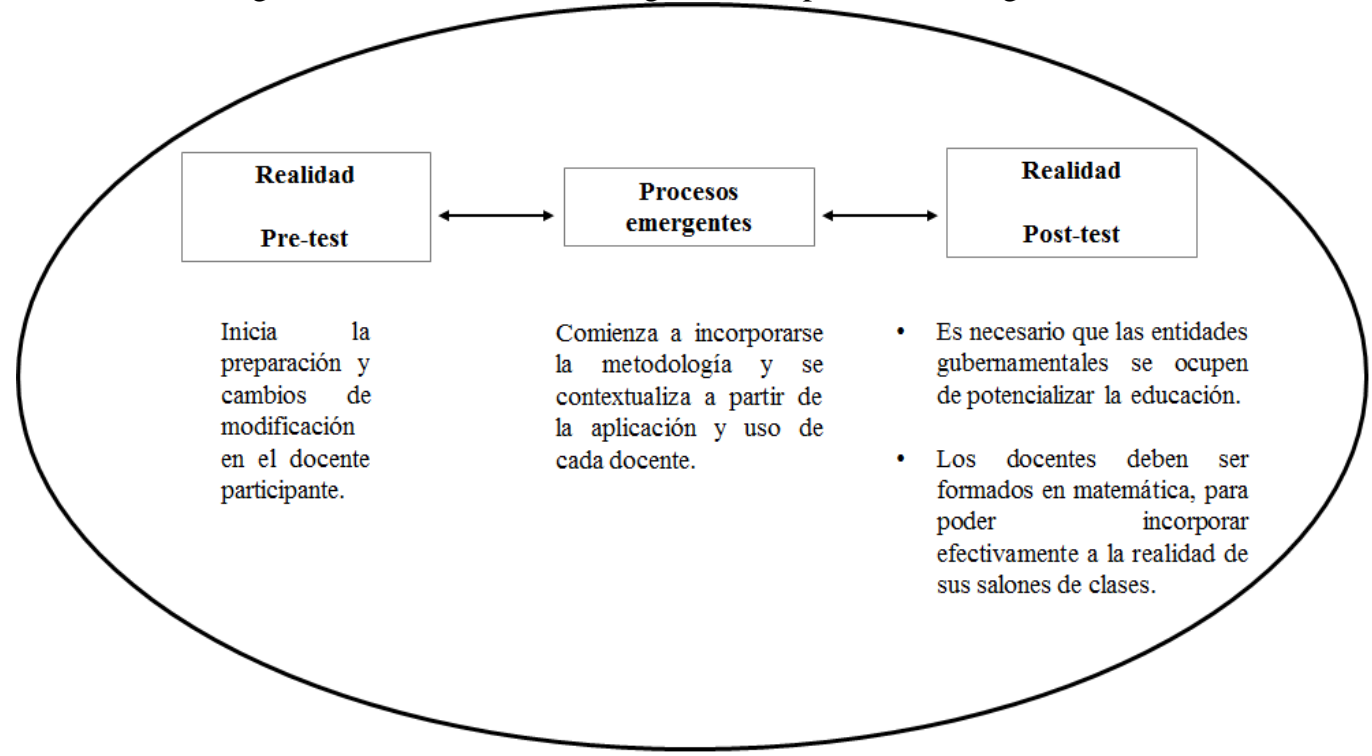

Se crearon actividades grupales con los profesores trabajando guías de actividades y su evaluación. Se construyeron guías de trabajo, material didáctico y evaluaciones formativas para los alumnos de las escuelas involucradas en el proyecto. Se distribuyeron al azar entre los profesores, diferentes actividades genéricas, de tal modo que cada grupo creó sus actividades y su evaluación, las cuales eran expuestas al final de la clase, con apoyo de computador y transparencias. Esta modalidad de trabajo se mantuvo durante toda la ejecución de la experiencia y estas acciones fueron registradas en su totalidad. Para el desarrollo del seguimiento, el estudio incluyó observaciones de clases.

El desarrollo de las sesiones se llevaron a cabo mediante el análisis y discusión de actividades didácticas, profundizando y analizando los contenidos matemáticos involucrados en éstas, así como sus implicaciones didácticas. Así mismo, se tuvo exposiciones por parte del instructor, se experimentaron actividades y se resolvieron problemas matemáticos. Para ello se implementaron estrategias de enseñanza tales como: exposición con interrogatorio, discusión grupal, trabajo por proyectos, demostración y resolución de actividades con y sin tecnología.

La experiencia que se vivió con los profesores de matemáticas, sugiere cuestionarse sobre el tipo de programas de formación en la que estos deben participar, así como el perfil deseado como consecuencia de estos programas, a continuación se presenta una tabla que resume las componentes en las que deberían enfocarse. 
Figura 4. Perfil del profesor de matemáticas encargado de diseñar tareas de aprendizaje

\begin{tabular}{|c|c|c|}
\hline Conocimientos matemáticos & $\begin{array}{c}\text { Elementos de educación } \\
\text { matemática }\end{array}$ & Uso de tecnologías digitales \\
\hline $\begin{array}{l}\text { - Conocimientos profundos } \\
\text { de los tópicos que aparecen } \\
\text { en el currículum de } \\
\text { matemáticas del nivel } \\
\text { escolar en que se desempeña. } \\
\text { - Habilidad para realizar } \\
\text { distintas formas de } \\
\text { representación de } \\
\text { información matemática. } \\
\text { - Saber mucho más de lo } \\
\text { que enseña, en cuanto a } \\
\text { tópicos de matemáticas se } \\
\text { refiere. } \\
\text { - Entender aspectos de otras } \\
\text { áreas del conocimiento como } \\
\text { química, biología o física, } \\
\text { que le permita establecer } \\
\text { relaciones con los tópicos de } \\
\text { matemáticas. }\end{array}$ & $\begin{array}{l}\text { - Conocimientos sobre } \\
\text { historia de las matemáticas. } \\
\text { - Saber acerca de los } \\
\text { principales aspectos de } \\
\text { algunas teorías sobre la } \\
\text { construcción del } \\
\text { conocimiento en los } \\
\text { individuos. } \\
\text { - Conocimiento sobre los } \\
\text { aspectos centrales de las } \\
\text { principales teorías, } \\
\text { perspectivas o marcos } \\
\text { teóricos sobre educación } \\
\text { matemática. }\end{array}$ & $\begin{array}{l}\text { - Conocimientos y habilidad } \\
\text { para manipular distintos } \\
\text { tipos de software para la } \\
\text { enseñanza de las } \\
\text { matemáticas. } \\
\text { - Conocimiento sobre } \\
\text { algunos aspectos de las } \\
\text { perspectivas teóricas que } \\
\text { estudian la forma en que un } \\
\text { individuo se apropia de un } \\
\text { instrumento para convertirlo } \\
\text { en una herramienta de } \\
\text { resolución de problemas. } \\
\text { - Apertura (Actitud) para } \\
\text { cambiar o modificar } \\
\text { aspectos metodológicos de } \\
\text { la forma en que imparte } \\
\text { clase. } \\
\text { - Interés investigativo para } \\
\text { buscar constantemente } \\
\text { nuevas formas de presentar } \\
\text { contenidos matemáticos con } \\
\text { el uso de la tecnología } \\
\text { digital }\end{array}$ \\
\hline
\end{tabular}

Fuente: Campos, 2010 b, p.78

\section{A modo de conclusión}

Se corroboró lo expuesto en diversas comunidades sobre el tema de la inteligencia emocional que incorporó entre otras, habilidades, desempeño docente y competencias, que establecen la conducta del profesor ante diversas interacciones. Se evidenció que los docentes en su inteligencia personal reflejan competencias que les permiten establecer formas de relacionarse con los otros, en este caso pares, estudiantes y comunidad educativa.

Como se evidenció que los docentes participantes en su inteligencia reflejaran competencias que les permitiera establecer habilidades, destrezas y potencialidades; básicamente, en la relación de persona a persona, lo que Vygotsky llama mediación (el aprendizaje por medio de signos sociales) y utilizando el desarrollo potencial de un aprendizaje ya adquirido para adquirir uno de mayor grado. Los docentes participantes establecían relaciones más asertivas en lo significativo y funcional. Aprendemos lo que nos es útil y, lo que no lo es, simplemente no nos interesa y lo desechamos.

Lo anterior, ha posibilitado reconocer que los docentes universitarios, establecen relaciones más asertivas. Además de identificar las habilidades 
para juzgarse a sí mismo, las limitaciones que posee y la sensibilidad hacia el aprendizaje. El discurso argumentativo del profesor habrá de enriquecerse ampliamente en la medida en que se incorporen aspectos tales como la resignificación de los saberes y la propia competencia de la argumentación (Rondero-Guerrero, Pérez, Acosta y otros, 2013, p. 51)

En la autorregulación o control de sí mismo, se destaca la habilidad para controlar las propias emociones e impulsos y canalizarlos hacia una meta. Estas competencias se veían reflejadas en los docentes participantes teniendo autocontrol ante diversas las experiencias, pues ellos eran capaces de realizar las asignaciones del facilitador sin experimentar cambios actitudinales.

Además, la automotivación les permitió estados de plenitud logrados en la medida que se alcanzaban las metas propuestas por el tutor designado en ambos seminarios, lo que implica una fijación en los objetivos por parte de ellos. Esto destaca competencias que determinan el modo en que se relacionan con los demás, en especial: empatía, habilidad para entender las necesidades, sentimientos y problemas de los demás, poniéndose en su lugar y responder correctamente a sus reacciones emocionales.

Los maestros empáticos, son capaces de escuchar a los demás, entender sus problemas, motivaciones, que normalmente tienen mucha popularidad y reconocimiento social, se anticipan a las necesidades de los demás y aprovechan las oportunidades que les ofrecen otras personas. La inteligencia emocional permite establecer relaciones con el otro, ejerciendo un liderazgo asertivo y proactivo. Por ello, los maestros en el contexto escolar al emplear su inteligencia emocional podrían orientar las emociones de sus alumnos desde los procesos de enseñanza-aprendizaje.

Existe consenso respecto al hecho de que para ser profesor de matemáticas, se debe por un lado, saber matemáticas y por otro lado saber enseñar matemáticas, sin embargo, como mencionan Ávila, Pérez y Santillán, (2008), lo primero no implica lo segundo. Para lograr ser un buen profesor de matemáticas, es indispensable la actualización constante en estas dos componentes.

Para que el profesor de matemáticas conozca la tecnología de que puede disponer y el uso que le puede dar, es necesario que participe en programas de formación y actualización permanentes, en los que además de profesores se deberán incluir a profesionales en educación matemática y en términos generales fomentarse el uso de la tecnología para el aprendizaje de las matemáticas. Este estudio tiene una tercera fase este año 2016, con patrocinio de la Secretaria Nacional de Ciencia Tecnología e Innovación (SENACYT), en la cual se pretende llegar a alcanzar una mayor comprensión del fenómeno de los procesos emergentes en el desarrollo de la inteligencia emocional a través de la resolución de problemas. 


\section{Acknowledgement:}

The Vice-Chancellorship for Research and Graduate Studies at the University of Panama by making payment of publication of this article.

\section{References:}

Ávila, A., Pérez, V. y Santillán, M. (2008). La formación de profesores de matemáticas en el CCH. En Barrera, F. et al. (Eds). Memorias del Segundo Seminario Nacional sobre Resolución de Problemas y el Aprendizaje de las Matemáticas. (pp. 59-65). Pachuca, Hidalgo, México.

Baptista, P., Fernández, C., \& Hernández, R. (2006). Metodología de la Investigación. México, Compañía.

Baron, R. (1997) Baron Emotional quotient inventory. Tecnical Manual. Toronto: Multi-health Systems Inc.

Barrantes, H. Mora, F (2008). ¿Qué es la Matemática? Creencias y Concepciones en la Enseñanza Media costarricense. En: Cuadernos de investigación y formación en educación matemática, número 4 pp. (71-81).

Berliner, D. C. (1986). In pursuit of the expert pedagogue. Educational researcher, 5-13.

Bisquerra, R. (2007). La autonomía emocional: status quaestionis de una controversia. Comunicació presentada en les III Jornades d'Educació Emocional: "Regulació Emocional i Convivència”.

Brophy, J. and Good T. (1986) Teacher Behavior and Student Achievement. In Handbook of Research on Teaching. 3erd ed. Edited by M. Wittrock pp. 328-375 New York: Macmillan.

Brophy, J. E. (1983). Research on the self-fulfilling prophecy and teacher expectations. Journal of educational psychology, 75(5), 631.

Campos, M. (2010, a). Formación de profesores y uso de tecnología en la educación matemática. En: Sepúlveda, L. A., García, P. R., Santos, T. L. M., Barrera, M. F., Sánchez, R. A. (Eds.).(2010). Memorias. XVIII Encuentro de Profesores de Matemáticas, III Seminario Nacional sobre Resolución de Problemas y el Aprendizaje de las Matemáticas, Morelia: UMSN.

Campos, M. (2010, b). Principios para el diseño de actividades de aprendizaje matemático con el uso de la tecnología. Tesis de Maestría no publicada. Universidad Autónoma del Estado de Hidalgo.

Cooper, R. K. y Sawaf, A. (1997). Executive EQ: Emotion intelligence in leadership and organizations. New York: Grosset Putnam.

Delors, J. (Coord.) (1996). La educación encierra un tesoro. Informe a la UNESCO de la Comisión Internacional sobre la educación para el siglo XXI. Madrid, España: Santillana. Ediciones UNESCO.

Frijda, N. H. (1994). Emotions are functional, most of the time. In P. Ekman and R. J. Davidson (Eds.). The nature of emotion: Fundamental questions (pp. 112-122). New York: Oxford University Press. 
Furnham, A., \& Petrides, K. V. (2003). Trait emotional intelligence and happiness. Social Behavior and Personality, 31, 815-823.

Gardner, H. (1983). Frames of Mind. New York: Basic Book Inc.

Godino, J. (2003). Funciones semióticas: un enfoque ontológico-semiótico de la cognición e instrucción matemática, Trabajo para optar a la Cátedra de Universidad de Didáctica de la matemática, España, Universidad de Granada, V, 5.

Goleman, D. (1995). Emotional intelligence. New York: Bantam Books.

Gómez Chacón, I. M. (2002) Cuestiones afectivas en la enseñanza de las matemáticas: una perspectiva para el profesor. In Aportaciones a la formación inicial de maestros en el área de matemáticas: una mirada a la práctica docente. Universidad de Extremadura: Cáceres, pp. 23-58.

Gómez-Chacón, I. M. (2000). Matemática emocional. Los afectos en el aprendizaje matemático. Madrid: Narcea.

LeDoux, J. E. (1996). The Emotional Brain: the Mysterious Underpinning of Emotional Life, Simon and Schuster, New York.

Maure, L. M., \& Marimon, G. (2014). Examining the role of college student's approach to math. Educational Research and Reviews, 9(19), 761770 .

Maya, J. Gatica, M. Hernández, Y. Morales, N. (2001). La andragogía aplicada en la capacitación de la NOM 087-ECOL-95. En: Rev Enferm IMSS 2001; 9 (2): 85-89.

Mayer, J. D., Salovey, P. (1997). “What is emotional intelligence?” En P. Salovey y D. Sluyter (Eds). Emotional Development and Emotional Intelligence: Implications for Educators (pp. 3-31). New York: Basic Books. Mayer, J. D., Salovey, P., \& Caruso, D. R. (2002). Mayer- Salovey-Caruso Emotional Intelligence Test (MSCEIT) item booklet. Toronto, Ontario, Canada: MHS Publishers.

Mayer, J.D. y Salovey, P. (1997). What is emotional intelligence? In P. Salovey \& D. Sluyter (Eds). Emotional Development and Emotional Intelligence: Implications for Educators (p. 3-31) Nueva York: Basic Books. Montoya, A. \& Velázquez, O. (sin año de publicación). Programa de formación profesional para instructores. Instituto Tecnológico de Sonora, México.

Pólya, G. (2001). Cómo plantear y resolver problemas (reimpresión de la $1^{\mathrm{a}}$ edición en español, 1965). México: Editorial Trillas.

Red Tercer Milenio (Sin año de publicación). Diseño e Impartición de Cursos de Capacitación. Manual del Participante. Consultado en línea: http://www.red3m.com.mx/formatos/manual_participante.pdf el día 20 de Octubre de 2011. 
Rondero-Guerrero, C., Perez, A., Acosta, J., Taasenko, A., Karelin, O. (2013). La formación de profesores en competencias matemáticas. México: Díaz de Santos.

Schutte, N.S., Malouff, J.M., Hall, L.E., Haggerty, D.J., Cooper, J.T., Golden, C.J., et al. (1998). Development and validation of a measure of emotional intelligence. Personality and Individual Differences, 25, 167-177. Shapiro, L. E. (1997). La inteligencia emocional de los niños. México: Javier Vergara

Simmons, S. y Simmons, J. C. (1997), Mesasuring Emotional Intelligence. Arlington (Texas): The Summit Publishing Group (trad. Cast. EQ. Cómo medir la inteligencia emocional. Madrid: Edaf, 1999)

Travería, G. T. (2008). El nuevo perfil profesional de los profesores de secundaria. Educación xx1, 11(1).

UNESCO-TERCE (2015). Factores Asociados al Logro de los Estudiantes de América Latina y el Caribe. Santiago: Salesianos Editores.

Wechsler, D. (1940). No intellective factors in general intelligence. Psychological Bulletin, 37, 444-445. 